\title{
PRODUÇÃO DE CATALISADOR MISTO Fe/Ti/Cu E USO EM PROCESSOS OXIDATIVOS AVANÇADOS
}

\author{
NOBREGA, E.S., SILVA, J.A., MADEIRA, V.S., SANTOS, G.B., FREITAS, A.E.R. \\ Universidade Federal da Paraíba, Departamento de Engenharia Química. \\ E-mail para contato: eltonobrega@ hotmail.com
}

\begin{abstract}
RESUMO - Neste trabalho, um catalisador sólido contendo $\mathrm{Fe} / \mathrm{Ti} / \mathrm{Cu}$ foi produzido pelo método Pechini, caracterizado e avaliado o seu uso na Fotocatálise Solar e na reação Fenton (para a degradação de Azul de Metileno). Os experimentos de Fotocatálise Solar mostraram que a mistura dos três semicondutores não foi eficiente para a Foto ativação dos sólidos, uma vez que o catalisador não apresentou eficiência na Fotocatálise. Com a adição de peróxido de hidrogênio, (reação do tipo Fenton), foi observada a degradação do corante, sendo a amostra que apresentou a maior eficiência aquela calcinada a $450^{\circ} \mathrm{C}$ por 1hora. Foram realizados experimentos variando-se a concentração de $\mathrm{H}_{2} \mathrm{O}_{2}$ e o pH da solução aquosa. Foi utilizado o modelo de Langmuir-Hinshelwood para ajustar a velocidade da reação, com coeficiente de correlação igual 0,7728 , constante intrínseca da reação, $\mathrm{k}_{1}$, igual a $0,0003(\mathrm{~L} / \mathrm{mg}) / \mathrm{min}$ e constante de equilíbrio $\left(\mathrm{K}_{2}\right)$ para adsorção de $\mathrm{H}_{2} \mathrm{O}_{2}$ igual a $0,090 \mathrm{~L} / \mathrm{mg}$. Com relação ao $\mathrm{pH}$, a amostra obteve maior eficiência na degradação do corante para o menor $\mathrm{pH}$ avaliado.
\end{abstract}

\section{INTRODUÇÃO}

A Fotocatálise é um Processo de Oxidação Avançada (POA) cujo princípio envolve a ativação por luz solar ou artificial de um sólido semicondutor, pela absorção de fótons com energia superior à energia de band gap, resultando na promoção de um elétron da banda de valência para a banda de condução com geração concomitante de uma lacuna $(\mathrm{h}+)$ na banda de valência. Essas lacunas mostram potenciais bastante positivos, e ao reagir com moléculas de água ou íons hidroxila podem gerar o radical hidroxila $\left(\mathrm{OH}^{*}\right)$, que é um forte agente oxidante, $\left(\mathrm{E}^{0}\left(\mathrm{OH}^{*} / \mathrm{H}_{2} \mathrm{O}\right)=+2,8 \mathrm{~V}_{\mathrm{NHE}}\right)$ (BOKARE e CHOI, 2014).

O sistema Fenton, homogêneo ou heterogêneo, é outro POA cuja degradação do composto orgânico é alcançada pelo ataque do radical hidroxila, que é gerado através da reação do $\mathrm{Fe}^{+2}$ com o $\mathrm{H}_{2} \mathrm{O}_{2}$ (Eqs. (1) e (2)). Em pH baixo, o $\mathrm{Fe}^{+3}$ solúvel, é reduzido a $\mathrm{Fe}^{+2}$, via $\mathrm{H}_{2} \mathrm{O}_{2}$, fechando desta maneira um ciclo catalítico $\mathrm{Fe}^{+2} / \mathrm{Fe}^{+3}$ (Eq. (3)). Como a velocidade da reação de oxidação do $\mathrm{Fe}^{+2}$ para $\mathrm{Fe}^{+3}$ é muito mais rápida $\left(\mathrm{k}_{1}>\mathrm{k}_{3}\right)$, em qualquer instante de tempo, há, no meio, um excesso de $\mathrm{Fe}^{+3}$, frente ao $\mathrm{Fe}^{+2}$. Um sistema catalítico mais eficiente, deve aumentar a velocidade da redução de $\mathrm{Fe}^{+3}$ para $\mathrm{Fe}^{+2}$, regenerando a espécie reduzida, para a reação Fenton. A irradiação de luz UV, no processo Foto-Fenton atinge este objetivo, com a Foto-redução do $\mathrm{Fe}^{+3}$ para $\mathrm{Fe}^{+2}$ e geração adicional de radical hidroxila (Eq. (4)) (DU et al., 2014). Os produtos finais das reações, considerando-se a completa mineralização dos contaminantes orgânicos, são $\mathrm{CO}_{2}$, água e ácidos inorgânicos. (Eq. (14))

$$
\mathrm{Fe}^{+2}+\mathrm{H}_{2} \mathrm{O}_{2} \stackrel{k_{1}}{\longrightarrow} \mathrm{Fe}^{+3}+\mathrm{OH}^{-}+\mathrm{OH}^{*} \quad k_{1}=51 \mathrm{M}^{-1} \mathrm{~s}^{-1}
$$




\section{Congresso Brasileiro de Engenharia Química em Iniciação Científica UFSCar - São Carlos - SP

$$
\begin{aligned}
& \mathrm{OH}^{*}+\mathrm{RH} \stackrel{k_{2}}{\longrightarrow} \mathrm{H}_{2} \mathrm{O}+\mathrm{R}^{*}(\text { radical orgânico }) \\
& \mathrm{H}_{2} \mathrm{O}_{2}+\mathrm{Fe}^{+3} \stackrel{k_{3}}{\longrightarrow} \mathrm{Fe}^{+2}+\mathrm{OOH}^{*}+H^{+} \quad k_{3}=0.001-0.01 M^{-1} s^{-1} \\
& \mathrm{Fe}^{+3} \mathrm{OH}^{-} \stackrel{\mathrm{Luz}}{\longrightarrow} \mathrm{Fe}_{s}^{+2}+\mathrm{OH}^{*}
\end{aligned}
$$

Um estudo recente realizado por nosso grupo de pesquisa mostrou que no sistema Foto Fenton Heterogêneo Solar o catalisador sólido $\mathrm{Fe}_{2} \mathrm{O}_{3} / \mathrm{TiO}_{2}$, produzido na proporção de $60: 40$ (\% em massa), apresentou eficiência e velocidade de degradação do corante azul de metileno, muito superior aquelas obtidas para os dois semicondutores quando isolados $\left(\mathrm{Fe}_{2} \mathrm{O}_{3}\right.$ ou $\left.\mathrm{TiO}_{2}\right)$. Isto sugere que a interação entre o Fe/Ti no catalisador atuou aumentando a velocidade da redução de $\mathrm{Fe}^{+3}$ para $\mathrm{Fe}^{+2}$, regenerando a espécie reduzida, para a reação Fenton (RAMALHO et al., 2017).

Processos denominados "Fenton-like systems" se baseiam no uso de outros metais que possuam múltiplos estados de oxidação, que ao reagir com o peróxido de hidrogênio, em um ciclo redox, também geram radicais hidroxila (BOKARE e CHOI, 2014). A atividade fotocatalítica de sistemas $\mathrm{Cu} / \mathrm{H}_{2} \mathrm{O}_{2}$ mostra que ambos os estados de oxidação do cobre $\left(\mathrm{Cu}^{+2} / \mathrm{Cu}^{+1}\right)$ reagem facilmente com peróxido de hidrogênio e complexos formados entre $\mathrm{Cu}^{2+}$ e intermediários orgânicos são facilmente decompostos por radicais hidroxila. Tais vantagens fazem com que o Cobre seja um candidato promissor no uso de sistemas do tipo Fenton (BOKARE e CHOI, 2014; SALAZAR et al., 2012).

Neste trabalho buscou-se, portanto, alcançar um efeito sinérgico entre o $\mathrm{Fe}, \mathrm{Ti}$ e $\mathrm{Cu}$, produzindo um catalisador à base de $\mathrm{Fe} / \mathrm{Ti} / \mathrm{Cu}$, na proporção 33,3:33,3:33,3 (\% em massa) para uso em tratamento de efluentes. Avaliou-se a eficiência deste catalisador na degradação do corante azul de metileno, por Fotocatálise Solar e reação Foto-Fenton Solar.

\section{PROCEDIMENTO}

\subsection{Produção do catalisador}

A síntese dos catalisadores foi por um método Pechini modificado (DANKS et al, 2016). Em um béquer de $1 \mathrm{~L}$, foram adicionados $600 \mathrm{~mL}$ de água destilada e mantido aquecimento até $\pm 80^{\circ} \mathrm{C}$. O ácido cítrico, na razão Ácido Cítrico/Cátion metálico de 3:1, foi adicionado ao béquer, sob agitação vigorosa, seguido do sulfato ferroso e nitrato de cobre. A adição do isopropóxido de titânio foi feita de forma suave mantendo-se a temperatura entre $70^{\circ} \mathrm{C}$ e $80^{\circ} \mathrm{C}$. A reação foi mantida sob aquecimento e agitação por $24 \mathrm{~h}$. Após isto, o etilenoglicol foi adicionado e a temperatura da reação foi elevada para $\pm 110^{\circ} \mathrm{C}$. Atingindo-se um ponto de viscosidade elevada (resina polimérica), a reação foi finalizada e a amostra pirolisada a $400^{\circ} \mathrm{C}$ por $1 \mathrm{~h}$. O material foi desaglomerado, peneirado (malha ABNT n ${ }^{\circ} 200$ mesh), e calcinado a $400^{\circ} \mathrm{C}(\mathrm{CU} .1), 450^{\circ} \mathrm{C}(\mathrm{CU} .2)$ e $500^{\circ} \mathrm{C}(\mathrm{CU} .3)$ por $1 \mathrm{~h}$.

\subsection{Uso do catalisador na degradação do corante azul de metileno}

Os ensaios para avaliar a atividade catalítica das amostras foram realizados em batelada, sob irradiação solar, no período das 10:00 até as 14:00 horas. Em um ensaio típico, $500 \mathrm{~mL}$ da solução aquosa, contendo $50 \mathrm{mg} / \mathrm{L}$ do corante, foram submetidos ao contato de $0,05 \mathrm{~g}$ do catalisador $(\mathrm{Fe} / \mathrm{Ti} / \mathrm{Cu})$, com introdução de $0,5 \mathrm{~L} / \mathrm{min}$ de ar. Inicialmente foram realizados 
experimentos variando-se as amostras do catalisador produzido (CU.1, CU.2 e CU.3) e a adição ou não de $\mathrm{H}_{2} \mathrm{O}_{2}$. Selecionada a melhor amostra (CU.2), bem como o POA aplicado (Foto-Fenton), variou-se a concentração de peróxido de hidrogênio e o pH inicial da solução aquosa. Alíquotas foram retiradas em intervalos pré-definidos, centrifugadas e analisadas em um espectrofotômetro de UV-vis (Shimadzu-1280) no comprimento de onda $665 \mathrm{~nm}$.

\section{RESULTADOS E DISCUSSÕES}

\subsection{Caracterização das amostras produzidas - DRX}

A Figura 1 ilustra o difratograma de raios $\mathrm{X}$ do sistema $\mathrm{Fe} / \mathrm{Ti} / \mathrm{Cu}$ obtido pelo método Pechini. As três amostras apresentaram baixa cristalinidade. A presença de picos característicos da estrutura da fase rutilo do $\mathrm{TiO}_{2}$ (JCPDS $n^{\circ} 76-1941$ ), hematita $\alpha-\mathrm{Fe}_{2} \mathrm{O}_{3}$ (JCPDS $n^{\circ}$ 79-1741), Sulfato de Cobre Hidratado (JCPDS $n^{\circ}$ 12-0782) e a Pseudobrookite $\mathrm{Fe}_{2} \mathrm{TiO}_{5}$ (JCPDS $\mathrm{n}^{\circ}$ 76-1158), foram evidenciadas, principalmente para as amostras calcinadas a $450^{\circ} \mathrm{C}$ (picos principais $2 \theta=12.1344^{\circ}, 27.2322^{\circ}$ e $31.3023^{\circ}$ ) e $500^{\circ} \mathrm{C}$ (picos principais $2 \theta=27,2126^{\circ}, 31,3299^{\circ}$ e 32,6250$)$.

Figura 1 - DRX das amostras CU.1, CU.2 e CU.3

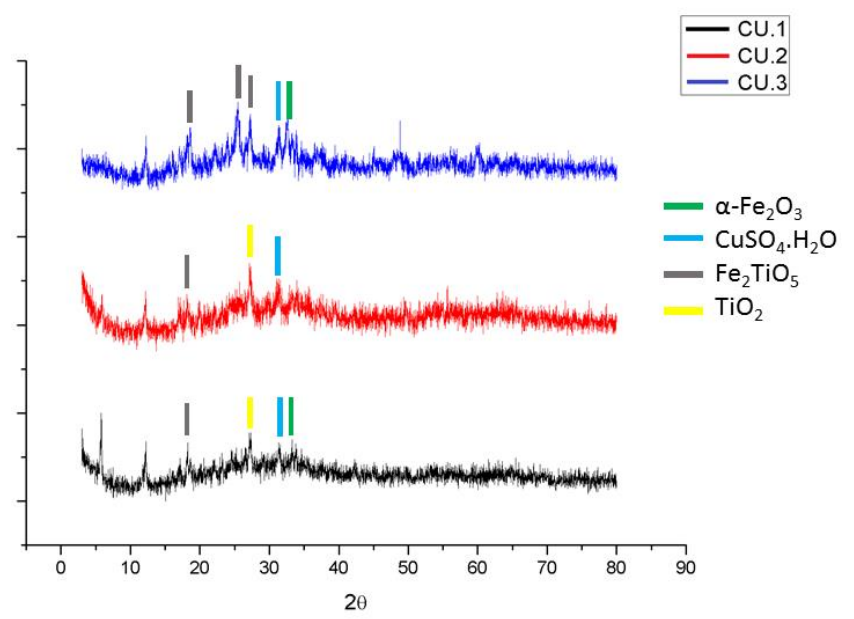

\subsection{Ensaios preliminares}

Foram realizados experimentos com todas as amostras do catalisador produzido e com a adição ou não de $\mathrm{H}_{2} \mathrm{O}_{2}$ (reação Foto-Fenton ou Fotocatálise, respectivamente). Para os dois experimentos a concentração inicial de azul de metileno foi de $50 \mathrm{mg} / \mathrm{L}$, o pH inicial da solução igual a 6,0 , a concentração de catalisador $0,1 \mathrm{~g} / \mathrm{L}$, a vazão de ar $0,5 \mathrm{~L} / \mathrm{min}$ e o branco é a solução do corante sem a adição do catalisador. Na figura 1 (a) vemos que a degradação do corante inicialmente é lenta e após 50min, há um pico de remoção, atingindo 35\% de remoção da cor para o branco. Isto sugere que a remoção do corante se dá por Fotólise, que o catalisador não apresenta eficiência na Fotocatálise Solar, e que não adsorve azul de metileno. A presença dos sólidos nas soluções impede a penetração da luz no meio obtendo menores taxas de remoção. O perfil das curvas cinéticas obtidas está relacionado diretamente com a incidência da irradiação solar no dia do experimento: inicialmente nublado e depois aberto. Embora haja dificuldades em se trabalhar nas condições ambientais, isso é justificado para se 
avaliar a real situação do processo em desenvolvimento (POA Solar). No segundo experimento, foi adicionado $10 \mathrm{mg} / \mathrm{L}$ de $\mathrm{H}_{2} \mathrm{O}_{2}$, em todas as soluções (inclusive no branco). $\mathrm{Na}$ figura 1 (b), observa-se uma mesma taxa inicial de remoção, para todas as amostras, (associada a Fotólise), e depois uma lenta degradação do corante somente para as amostras contendo o sólido (Foto-Fenton). A amostra CU.2 apresentou a maior eficiência, cerca de $45 \%$ de remoção com $10 \mathrm{mg} / \mathrm{L}$ de $\mathrm{H}_{2} \mathrm{O}_{2}$. A adsorção prévia de $\mathrm{H}_{2} \mathrm{O}_{2}$ e reações redox entre as espécies metálicas $(\mathrm{Fe} / \mathrm{Ti} / \mathrm{Cu})$, podem estar causando o retardo inicial da reação.

Figura 1 - Ensaios preliminares. (a) Sem peróxido de hidrogênio - Fotocatálise. (b) Com peróxido de hidrogênio - Foto-Fenton.

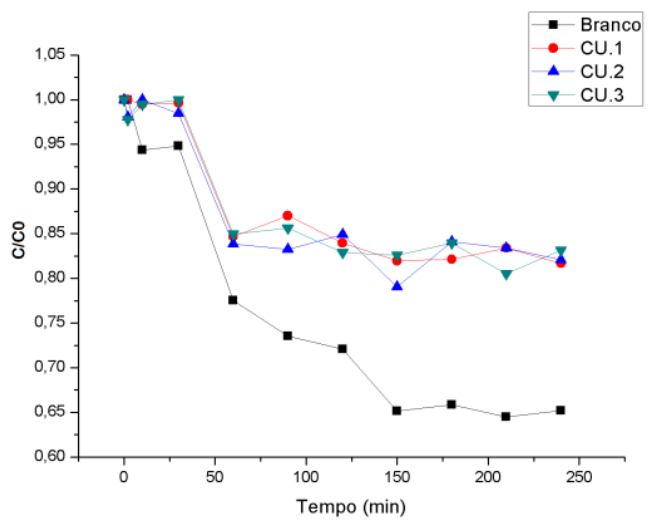

(a)

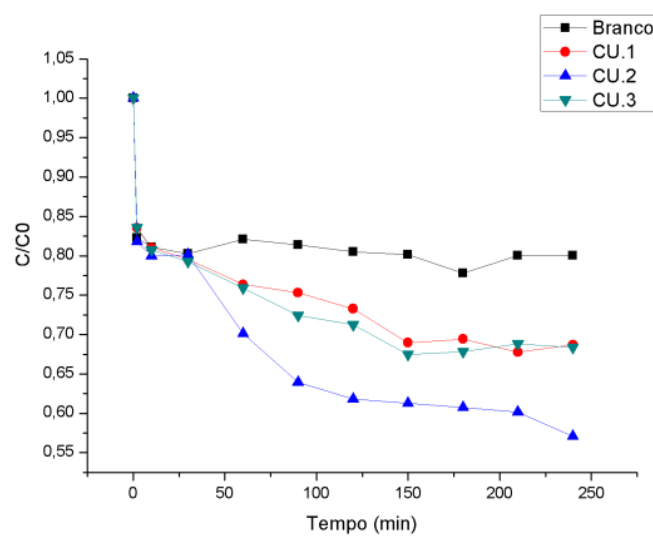

(b)

\subsection{Avaliação do Processo Foto-Fenton}

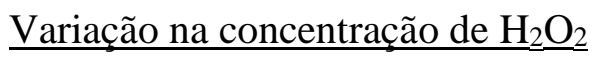

Utilizando a amostra de CU.2, variou-se a concentração de $\mathrm{H}_{2} \mathrm{O}_{2}$, na faixa de 5 até $50 \mathrm{mg} / \mathrm{L}$, mantendo-se constante os demais parâmetros. Os resultados na figura 2 (a), indicam que uma concentração de $\mathrm{H}_{2} \mathrm{O}_{2}$ na faixa de $30 \mathrm{mg} / \mathrm{L}$, é suficiente para se obter entre 60 e $70 \%$ de remoção da cor. Plotando-se a velocidade inicial da reação em função da concentração inicial de $\mathrm{H}_{2} \mathrm{O}_{2}$, observa-se que para baixas concentrações, a velocidade da reação é linear com a mesma, e para altas concentrações se torna aproximadamente constante, figura 2 (b). $\mathrm{O}$ modelo de Langmuir-Hinshelwood, foi utilizado para ajustar a lei de velocidade da reação, cujos valores do coeficiente de correlação - $\mathrm{R}^{2}$, constante intrínseca da reação - $\mathrm{k}_{1}$ e constante de equilíbrio para adsorção de $\mathrm{H}_{2} \mathrm{O}_{2}-\mathrm{K}_{2}$, estão listados na Tabela 1. O ajuste realizado não foi satisfatório, $\left(\mathrm{R}^{2}=0,77\right)$, e é possível observar que a introdução de cobre no catalisador não surtiu o efeito desejado, quando comparado com o catalisador previamente produzido por nosso grupo de pesquisa $\left(\mathrm{Fe}_{2} \mathrm{O}_{3} / \mathrm{TiO}_{2}-60 / 40\right)$. Estudos revelaram que o $\mathrm{Cu}^{+}$reage com o oxigênio formando $\mathrm{Cu}^{+2}$ (BOKARE e CHOI, 2014), o que pode retardar a degradação do corante. A oscilação na concentração de azul de metileno obtida no experimento pode estar 
relacionada com reações paralelas de lixiviação dos metais para a fase líquida, formação de hidróxidos metálicos (que tem capacidade de adsorção) e reações do tipo Fenton homogênea.

Figura 2 - Foto-Fenton com a amostra CU.2 variando-se a concentração de $\mathrm{H}_{2} \mathrm{O}_{2}$. (a) Cinética de remoção de cor (b) Velocidade inicial da reação versus concentração inicial de $\mathrm{H}_{2} \mathrm{O}_{2}$.

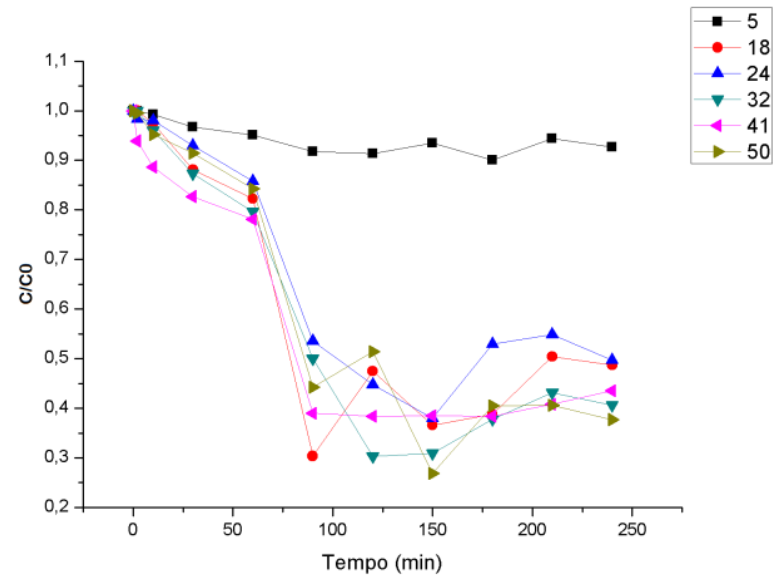

(a)

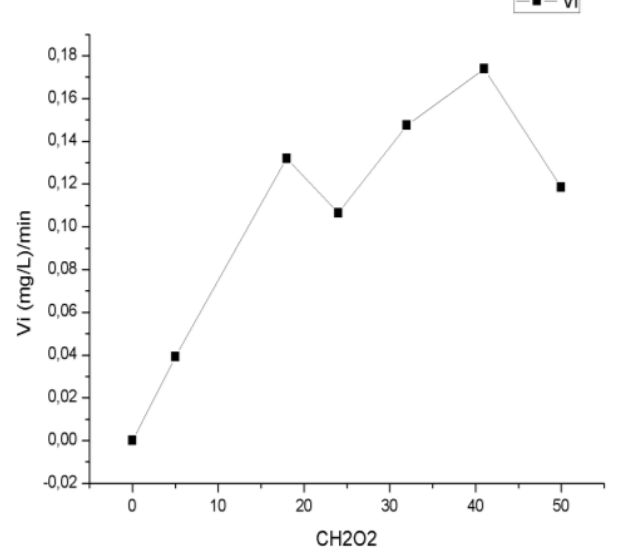

(b)

Tabela 1 - Comparação com um trabalho anterior do mesmo grupo de pesquisa

\begin{tabular}{|c|c|c|c|c|}
\hline Amostra & Referência & $\mathrm{R}^{2(1)}$ & $\mathrm{k}_{1}{ }^{(2)},(\mathrm{L} / \mathrm{mg}) / \mathrm{min}$ & $\mathrm{K}_{2}{ }^{(3)}, \mathrm{L} / \mathrm{mg}$ \\
\hline $\mathrm{Fe}_{2} \mathrm{O}_{3} / \mathrm{TiO}_{2} / \mathrm{CuO}$ & Este trabalho & 0.7728 & 0.0003 & 0.090 \\
\hline $\mathrm{Fe}_{2} \mathrm{O}_{3} / \mathrm{TiO}_{2}$ & RAMALHO et al., 2017 & 0,9941 & 0,0700 & 0,140 \\
\hline
\end{tabular}

(1) Coeficiente de correlação, (2) Constante intrínseca, (3) Constante de equilíbrio.

\section{Variação no pH da solução aquosa:}

Utilizando a concentração de $\mathrm{H}_{2} \mathrm{O}_{2}$ definida anteriormente, $32 \mathrm{mg} / \mathrm{L}$, variou-se o $\mathrm{pH}$ da solução aquosa na faixa de 3,0 até 10,5, mantendo-se os demais parâmetros constantes. Os resultados obtidos (figura 3), mostram que quanto menor o $\mathrm{pH}$ da solução aquosa, maior a remoção de cor. $\mathrm{O}$ pH ótimo, no qual se obteve $96 \%$ de degradação do corante, foi 3,0. O aumento da concentração de corante nas amostras com pHs mais elevados pode estar associado a dependência da cor com o pH.

Com base nos resultados dos experimentos, propõem-se um mecanismo para a reação FotoFenton Solar com o uso de $\mathrm{Fe} / \mathrm{Ti} / \mathrm{Cu}$. O processo inicia-se com a adsorção de $\mathrm{H}_{2} \mathrm{O}_{2}$ na superfície do sólido; Reação na superfície com as espécies metálicas, obtendo-se as espécies reduzidas e redução no pH do meio; Lixiviação de cobre (alternativamente ferro), para a fase líquida; Reações paralelas de precipitação e solubilização (com adsorção/dessorção) versus reação do tipo Fenton homogênea (reações 8 e 9); Degradação do corante via radical hidroxil (reações 12 e 13).

Figura 3 - (a) Mecanismos de reação para remoção de corante, (b) Ensaio realizado com diferntes pHs. 


$$
\begin{aligned}
& \mathrm{H}_{2} \mathrm{O}_{2}+\mathrm{S} \leftrightarrow \mathrm{H}_{2} \mathrm{O}_{2} \cdot \mathrm{S} \\
& \mathrm{H}_{2} \mathrm{O}_{2} \mathrm{~S}+\mathrm{Fe} / \mathrm{Cu} / \mathrm{Ti} \rightarrow \mathrm{OOH}^{*} \mathrm{~S}+\mathrm{H}^{+}+\mathrm{Fe}^{+2} / \mathrm{Cu} / \mathrm{Ti} \\
& \mathrm{H}_{2} \mathrm{O}_{2} \mathrm{~S}+\mathrm{Fe} / \mathrm{Cu} / \mathrm{Ti} \rightarrow \mathrm{OOH}^{*} \cdot \mathrm{S}+\mathrm{H}^{+}+\mathrm{Fe} / \mathrm{Cu}^{+1} / \mathrm{Ti} \\
& \mathrm{Fe} / \mathrm{Cu} / \mathrm{Ti} \stackrel{{ }^{2} \mathrm{H}}{\longrightarrow} \mathrm{Cu}^{+2}(\mathrm{l}) \text { ou } \mathrm{Fe}^{+2}(i) \\
& \mathrm{Cu}_{(\mathrm{l})}^{+2}+2 \mathrm{OH}^{-} \leftrightarrow \underbrace{\mathrm{Cu}(\mathrm{OH})_{2}}_{S} \\
& S^{\prime}+A M \leftrightarrow A M \cdot S^{\prime} \\
& \mathrm{Cu}_{(i)}^{+2}+\mathrm{H}_{2} \mathrm{O}_{2(i)} \rightarrow \mathrm{OOH}^{*}+\mathrm{H}^{+}+\mathrm{Cu}^{+1} \\
& \mathrm{Cu}_{(\mathrm{l})}^{+1}+\mathrm{H}_{2} \mathrm{O}_{2(\mathrm{l})} \rightarrow \mathrm{OH}^{*}+\mathrm{OH}^{-}+\mathrm{Cu}^{+2} \\
& \mathrm{OH}_{(())}^{*}+\mathrm{AM}_{(\mathrm{i})} \rightarrow \mathrm{AM}^{*}+\mathrm{H}_{2} \mathrm{O} \\
& \mathrm{O}_{2(i)}+\mathrm{AM}_{(())} \rightarrow \mathrm{CO}_{2}+\mathrm{H}_{2} \mathrm{O}+\text { ácidos }
\end{aligned}
$$

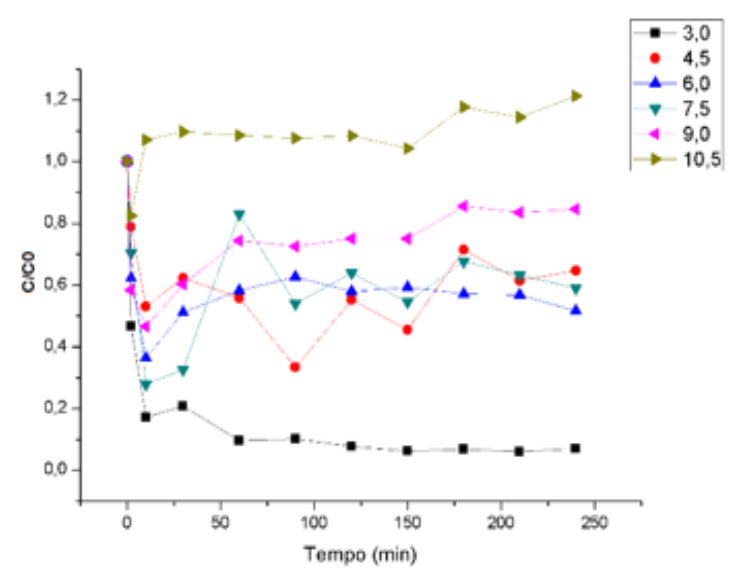

(b)

\section{CONCLUSÃO}

Foi produzido um catalisador sólido de $\mathrm{Fe} / \mathrm{Ti} / \mathrm{Cu}$, na mesma proporção em massa por um método Pechini modificado. As amostras produzidas apresentaram baixa cristalinidade, mas foi observada a presença de picos característicos da hematita $\left(\alpha-\mathrm{Fe}_{2} \mathrm{O}_{3}\right)$, do $\mathrm{TiO}_{2}$ na fase rutilo e da pseudobrookita $\left(\mathrm{Fe}_{2} \mathrm{TiO}_{5}\right)$. O catalisador não apresentou eficiência na Fotocatálise Solar, entretanto, na reação Foto-Fenton Solar foi alcançada $96 \%$ de degradação do corante, em aproximadamente 60 minutos de reação, partindo-se de uma solução aquosa com $50 \mathrm{mg} / \mathrm{L}$ de azul de metileno, 0,1g sólido/L, 32mg/L de $\mathrm{H}_{2} \mathrm{O}_{2}$ e $\mathrm{pH} 3,0$. Foi utilizado o modelo de Langmuir-Hinshelwood para ajustar a velocidade da reação, com coeficiente de correlação igual 0.7728 , constante intrínseca da reação, $\mathrm{k}_{1}$, igual a $0.0003(\mathrm{~L} / \mathrm{mg}) / \mathrm{min}$ e constante de equilíbrio $\left(\mathrm{K}_{2}\right)$ para adsorção de $\mathrm{H}_{2} \mathrm{O}_{2}$ igual a $0.090 \mathrm{~L} / \mathrm{mg}$. Ensaios avaliando-se a influência da adição de oxigênio na mistura, a variação da concentração de azul de metileno e de sólidos, bem como a lixiviação dos metais para a solução aquosa, estão sendo realizados.

\section{REFERÊNCIAS}

BOKARE, A.D.; CHOI, W. Review of iron-free Fenton-like systems for activating $\mathrm{H} 2 \mathrm{O} 2$ in oxidation processes. Journal of Hazardous Materials, V. 275, p.121-135, 2014.

DANKS, A. E; HALL, S. R.; SCHNEPP, Z. The evolution of 'sol-gel' chemistry as a technique for materials synthesis. A Material Horizons., V. 3, p. 91-112, 2016.

DU, Y.; ZHAO, L.; ZHANG, Y. Roles of TaON and $\mathrm{Ta}_{3} \mathrm{~N}_{5}$ in the visible-Fento-like degradation of atrazine. Journal of Hazardous Materials 267, 55-61, 2014.

RAMALHO, M. L. R. A.; MADEIRA, V. S.; SILVA, J. A.; BRASILEIRO, I. L. O.; SILVA NETO, J. S. Heterojunction $\mathrm{Fe}_{2} \mathrm{O}_{3} / \mathrm{TiO}_{2}$ production and its use in solar photocatalysis. The 14th IWA Leading Edge Conference on Water and Wastewater Technologies, 2017, Florianópolis. The Internation Water Association Publishing, 2017.

SALAZAR, R.; BRILLAS, E.; SIRÉS, I. Finding the best $\mathrm{Fe}^{2+} / \mathrm{Cu}^{2+}$ combination for the solar photoelectron-Fenton treatment of simulated wastewater containing the industrial textile dye Disperse Blue 3. Applied Catalyst B: Environmental, 115-116, 107-116, 2012. 